\title{
PUBLISHED VERSION
}

Infante, Fernando A.; Proudfoot, Judith; Davies, Geoffrey Paul; Bubner, Tanya Kaye; Holton, Christine Helen; Beilby, Justin John; Harris, Mark F.

How people with chronic illnesses view their care in general practice: a qualitative study Medical Journal of Australia, 2004; 181(2):70-73

This article is available from the Medical Journal of Australia at:

https://www.mja.com.au/journal/2004/181/2/how-people-chronic-illnesses-view-their-caregeneral-practice-qualitative-study

PERMISSIONS

This document has been archived with permission from the editor of the Medical Journal of Australia, 26 April 2007. 


\section{GP IN ACTION - RESEARCH}

\section{How people with chronic illnesses view their care in general practice: a qualitative study}

Fernando A Infante, Judith G Proudfoot, Gawaine Powell Davies, Tanya K Bubner, Chris H Holton, Justin J Beilby and Mark F Harris

C hronic disease is a major challenge for general practice. Over $80 \%$ of the illness and $50 \%$ have two or more. ${ }^{1}$ Most care is provided within general practice, but its quality is variable, with only $50 \%$ of patients receiving optimum clinical care., ${ }^{2,3}$

There is growing interest in patients' perceptions of their care. In the United Kingdom, consumers have identified problems with access to care, consultation length, provision of information, communication and continuity of care. ${ }^{4}$ Expectations are high, particularly for continuity and access, and exceed UK government targets for general practice. ${ }^{5}$ A study across 10 European countries showed patients were generally happy with confidentiality, GPs' listening to patients, time available during consultations and urgent responses, but not about organisational aspects of care. ${ }^{6}$

In Australia, consultations by the Consumers' Health Forum between 1992 and $1999^{7}$ indicated that expectations of general practice included improved communication; more referral to health and support services; appropriate, timely and quality healthcare; accessible services; and better use of information technology. Australian consumers expect their GPs' undivided attention, the most up-to-date and relevant treatments and relevant information. ${ }^{8}$

However, there is little information about the specific expectations of consumers with chronic conditions. A national Consumers' Health Forum study found that consumers with special needs (diabetes, arthritis, HIV, AIDS, multiple sclerosis) valued an equal "partnership" of care between patient, GP, other healthcare professionals and carers, and sensitivity by the GP to the medical, social and psychological implications of the elderly have at least one chronic

\section{ABSTRACT}

Objectives: To explore the perceptions of patients with chronic conditions about the nature and quality of their care in general practice.

Design: Qualitative study using focus group methods conducted 1 June to 30 November 2002.

Participants and setting: 76 consumers in 12 focus groups in New South Wales and South Australia.

Main outcome measures: Recurring issues and themes on care received in general practice.

Results: Three groups of priorities emerged. One centred on the quality of doctors, including technical competence, interpersonal skills, time for the patient in the consultation and continuity of care. A second concerned the role of patients and consumer organisations, with patients wanting (i) recognition of their knowledge about their condition and self-management, and (ii) for GPs to develop closer links with consumer organisations and inform patients about them. The third focused on the practice team and the importance of practice nurses and receptionists.

Conclusion: GPs should consider the amount of time they spend with chronically ill patients, and their interpersonal skills and understanding of patients' needs. They need to be better informed about the benefits of patient self-management and consumer organisations, and to incorporate them into their care. They also need to review how their practice nurses and receptionists can maximise the care of patients.

MJA 2004; 181: 70-73 patient's medical condition. ${ }^{9}$ This is consistent with a South Australian study in which 10 patients with asthma and diabetes described difficulties with healthcare professionals knowing less than they did about their illness, not listening or taking time, or focusing on the condition rather than the person. ${ }^{10}$

We have previously reported GPs' views of chronic disease care in Australian general practice. ${ }^{11}$ A letter in response suggested that patients and GPs share the same goals for chronic disease management, but from a different perspective. ${ }^{12}$ In this article, we explore, specifically, the perceptions of patients with chronic conditions about
School of Public Health and Community Medicine, University of New South Wales, Sydney, NSW. Fernando A Infante, MPH, Research Associate; Judith G Proudfoot, PhD, MA, BEd(Hons), Senior Research Fellow; Gawaine Powell Davies, MHP, Centre director; Mark F Harris, DRACOG, FRACGP, MD, Professor of General Practice.

Department of General Practice, The University of Adelaide, Adelaide, SA.

Tanya K Bubner, BSocSc, Project Officer; Chris H Holton, GDPH, GDAcc, BA(Acc), Project Officer; Justin J Beilby, MD, MPH, Head of Department.

Reprints will not be available from the authors. Correspondence: Dr Judith G Proudfoot,

Centre for General Practice Integration Studies, School of Public Health and Community Medicine, University of New South Wales, Sydney, NSW 2052. j.proudfoot@unsw.edu.au chronic disease care in Australian general practice.

\section{METHODS}

We recruited patients with chronic conditions by advertising in a local (south-eastern) Sydney newspaper and in newsletters of Divisions of General Practice, and by approaching all relevant consumer organisations. We received positive responses from the Arthritis Foundation of NSW, Epilepsy NSW, Diabetes Australia, Parkinsons NSW, the Asthma Foundation, and local support groups in South Australia. People with diabetes, cardiovascular disease, asthma, depression or any other chronic illness were invited to participate.

Two researchers from the University of New South Wales and two from the University of Adelaide conducted 12 focus groups. Two researchers attended each focus group: one facilitated the discussion using a standardised approach and set of questions, and the other audiotaped the discussions, noting interesting non-verbal features. Each focus group lasted about 90 minutes. Topics were 
based on the literature and reflected those used in our GP focus groups. ${ }^{11}$ Questions focused on:

- desirable skills and qualities in a GP caring for patients with chronic diseases;

- characteristics of a good general practice; - continuity of care;

- access to other health professionals; and

- GPs' and practice staff's understanding of the point of view of patients with chronic conditions.

Participants were not paid, but were reimbursed for expenses. They received an information sheet and signed a consent form before participating. Audiotapes were transcribed and then erased. Ethical approval for the study was obtained from the ethics committees of the two universities.

\section{Analysis}

Transcripts were analysed using NUD*IST NVivo software ${ }^{13}$ and key themes extracted by one of us (FAI). An independent person performed a second (manual) analysis of the transcripts. The extracted themes were verified by J GP, and any differences resolved by discussion among FA I, J GP and the independent assessor.

\section{RESULTS}

Seventy-six health consumers participated in 12 focus groups (11 in New South Wales and one in South Australia) between 1 June and 30 November 2002. Demographic characteristics of the participants are shown in Box 1 .

Participants were older than average (average, 63 years) and most attended bulkbilling practices; $59 \%$ were female. Chronic conditions included arthritis, diabetes (types 1 and 2), cardiovascular disease, asthma, epilepsy, chronic obstructive pulmonary disease, depression and Parkinson's disease. Comorbidities were common (average, 2-3 conditions per participant).

The following key themes emerged.

1. Continuity of care: All focus groups emphasised the importance of a continuing relationship with a GP, giving patients confidence to express their needs and building trust. A young patient with asthma said: "It can take a long time to trust somebody when it's your life you're trusting them with ... to build up that relationship where you feel you can say: 'Well, hang on, what's this medication doing for me'."

Continuity of care was also considered important when a patient was admitted to hospital, or seen by another practitioner,

1 Demographic details of the 76 participating patients

\begin{tabular}{lr} 
Characteristic & Number \\
\hline Mean age in years (95\% Cl; range) & $63.4(60.1-66.8 ; 19-84)$ \\
Sex & $45(59 \%)$ \\
Female & $31(41 \%)$ \\
Male & $2.4(2.0-2.8 ; 1-10)$ \\
Mean number of chronic conditions per participant (95\% Cl; range) & $21.7(17.6-25.7 ; 1-65)$ \\
Mean no. of years of participants' longest-standing condition $(95 \%$ & \\
Cl; range) & $3.5(2.9-4.2 ; 1-13)$ \\
Mean no. of GPs in participants' GP practices (95\% Cl; range) & $52(71 \% ;$ missing, 3) \\
Participants who attend a bulk-billing practice & $16(22 \% ;$ missing, 4) \\
Participants who attend a practice which recalls patients for & 25 (34\%; missing, 3) \\
structured care & 20 (30\%, missing, 9) \\
Participants who attend a practice with a practice nurse & \\
Participants who attend a practice with a practice manager & $17(22.4 \%)$ \\
Recruitment method & $59(77.6 \%)$ \\
Local paper/Division of General Practice newsletter &
\end{tabular}

and having the medical history with one doctor saved time for both patient and GP. On the other hand, some participants believed GPs could become complacent seeing the same patients for years, and felt a second opinion could sometimes be useful.

Participants valued seeing their GPs regularly to check progress, even when they were not feeling ill. In most groups, participants viewed their GP as their main healthcare provider and care coordinator, although this sometimes changed as GPs moved from sole provider to member of a multidisciplinary team. Most participants were happy with their GPs, but would change practitioner if their needs were not being met, as several had already done.

2. GPs and specialists: A second strong theme in all groups was access to other healthcare providers. Opinions varied. Some found it relatively easy to get a referral from their GP to another healthcare professional, especially when GPs had a comprehensive network of trusted specialists. For these participants, negotiating appropriate specialist care had often been important in building the doctor-patient relationship. A few, however, had found it very difficult get a referral.

There was consensus across all the groups that a good relationship between GPs and specialists improved care, although one group thought GPs might lose interest in patients once they had been referred to a specialist.
3. Profile of a "good" GP: There was agreement about what made a good GP (Box 2). Interpersonal skills were considered at least as important as clinical skills, with some groups preferring a female doctor because of better interpersonal dynamics.

GPs' ability to communicate was considered extremely important. Participants in two groups indicated they would travel significant distances to attend a GP from their own cultural or ethnic background.

A "poor" GP, by contrast, was described as pressured, with too little time for patients. In 10 of the 12 groups, it was held that GPs could at times be impersonal and dismissive, particularly with the elderly, those with certain chronic conditions such as osteoarthritis, and stigmatised conditions like hepatitis $C$.

It was generally agreed that GPs were weak in recognising and treating the emotional impact of chronic illness: "They're good at the actual management of the condition itself, but the psychological side of how it affects you is more or less ignored."

Participants also discussed the importance of GPs' continuing professional development (CPD). They wanted to know that their GP was enrolled in some form of CPD, was up-to-date with medical knowledge and knew about new treatments. It was seen as a strength rather than a weakness if GPs consulted guidelines during the consultation. 4. Involvement of patients in their own chronic care: All groups debated whether patients should have an active role in the 


\begin{tabular}{ll}
2 Characteristics of a "good" general practitioner \\
Characteristic $\quad$ Features \\
\hline Interpersonal skills & Good listener \\
& Understanding, caring, empathic and compassionate \\
& Counsels; provides advice and support \\
& Communicates well with patients \\
& Trusts and believes patients; does not dismiss or ignore their concerns \\
& Respects patients and treats each as an individual \\
& Good diagnostician \\
& Knowledgable about the chronic condition, its course and its treatment \\
& Up-to-date \\
& Holistic approach, taking into account patients' overall health \\
& Able to guide patients through the different stages of their chronic \\
& conditions \\
& Provides patient education with emphasis on self-management \\
& Will refer patients when necessary, accepting that she or he does not \\
& know everything about the condition and may need help \\
& Is accessible on the telephone \\
& Has time for patients
\end{tabular}

3 Profile of a good general practice

\begin{tabular}{ll} 
Desirable features & Specific examples \\
\hline Clinical services & A variety of clinical services provided in addition to standard \\
& general practice services, including $\bullet$ x-ray imaging \\
& $\bullet$ pathology laboratory $\bullet$ electrocardiogram \\
Access to services and time & $\bullet$ Convenient consultation times (eg, after hours) $\bullet$ Home visits \\
management & - Short waiting times $\bullet$ Longer consultations \\
Staffing & - Bulk-billing $\bullet$ Good triage system \\
& - Has a practice nurse $\bullet$ Has a practice manager \\
Other facilities & $\bullet$ Friendly receptionists \\
& $\bullet$ Comfortable reception area $\bullet$ "Quiet" room for sicker \\
& patients $\bullet$ Computerised $\bullet$ Recall/reminder system in place
\end{tabular}

management of their condition. Some considered themselves as expert as their GP about their condition: "I want somebody who is going to treat me as a partner in the management of the condition, who's going to respect my opinion and realise that I'm managing it 24/7/365 and not just 9 to 5 Monday to Friday."

However, there was no consensus. In half the groups, some participants preferred their GP to take responsibility for their care, believing "It's the doctor's job to keep me healthy".

Most participants thought that GPs encouraged patients to take an active role in their care, although the view was expressed in two groups that some GPs actively discouraged this.

Participants discussed how much patients wanted to know about their condition.
Some considered too much initial information overwhelming, and suggested GPs should tailor information-giving to the stage of the patient's condition and their adaptation to it: "I think that the best that the GP can do is try and give guidance to the individual as they're going through the stages of their chronic condition."

Lifestyle changes for better self-care generated considerable discussion. In most groups, they were seen as important, but opinion was divided as to whether doctors provided enough education about this. Interestingly, a few felt that GPs overemphasised lifestyle as the cause of particular chronic diseases instead of looking for the "real" cause.

5. The role of consumer organisations: There was general agreement that consumer organisations or support groups were valua- ble. They served many roles, providing consumers with information, support, friendship and understanding, as well as being a resource for GPs. However, GPs were criticised in eight groups for not sufficiently informing patients about such organisations.

6. Profile of a good general practice: At the centre of any good practice, most groups agreed, is a good doctor: "It wouldn't matter whether they were working in a, you know, a falling-down building ... they don't necessarily need the greatest of facilities to be a good GP, and that's what I'd aim for, just a good GP."

Box 3 summarises other aspects of good practices. Time management was widely discussed, with waiting - for an appointment date and in the waiting room - a common complaint. However, some were willing to be flexible about time in the waiting room, provided reception staff kept them informed about delays. Participants in some groups also valued their GP's time and were careful not to extend consultations unnecessarily: "It seems like it's common in the practice that they devote however much time's necessary to each individual patient, which, you know, is a bit of a nuisance... but by the same token ... that's better medicine."

Receptionists were considered important because they usually knew the patients, could talk to them and help streamline waiting times by assessing the urgency of patients' needs for medical attention: "[receptionists] get to know you and you feel welcome because they address you by a name... and they understand that ... if you come in in a mad flap, there's something drastically wrong."

Practice nurses were also considered important, particularly for giving patients information about their condition and undertaking some parts of the care: "It was wonderful, because you could go and see the nurse, and she'd say: 'Oh right, well, you don't need to go and see X', so in you'd go and she'd take out your stitches, or whatever, which I think freed up... the GP's time a fair bit."

Large practices were valued for their extended hours, wide range of services and facilities, levels of bulk-billing and peer pressure to keep GPs up-to-date, but were considered sometimes impersonal. A high turnover of GPs in medical centres could undermine continuity and quality of care. Smaller practices were more likely to offer home visits, have friendlier staff and a GP who knew the patient better. 


\section{DISCUSSION}

We found common themes among a diverse sample of NSW and South Australian health consumers in their views about chronic disease care in Australian general practice. GPs were their main healthcare providers and coordinators of their care. They were concerned about GPs' clinical skills, but viewed interpersonal skills as equally important. The ongoing relationship between the doctor and patient was crucial. GPs were expected to know the medical side of a condition and also to understand its social and psychological impacts.

This is consistent with previous Australian research by the Consumers' Health Forum, which found consumers' perceptions of quality in general practice highly focused on doctors, especially technical competence and interpersonal skills. ${ }^{9}$ It is also consistent with international literature, which additionally highlights access to the GP, provision of information and adequate length of consultations. ${ }^{9}$ Our findings support the call for GPs to recognise patients' "subjective experience" of their condition in addition to its "biochemical parameters". ${ }^{14}$

However, our finding that some consumers considered they knew more than their GP about their condition adds complexity. They wanted their GP to recognise their expertise and work with them as partners in the management of their condition. Although the benefits of this type of partnership have been demonstrated, they are not accepted by all doctors. ${ }^{15,16}$ In our previous study of GPs' perceptions of chronic disease management, some GPs found it unrewarding to work with patients who could not be cured and were frustrated at levels of compliance with treatment. $^{11}$

Access to the practice was valued, as were reception staff (who could facilitate access, among other tasks) and practice nurses. This is consistent with overseas findings, which highlight the role of professionals other than GPs in the care of patients with chronic disease. ${ }^{17}$

Our study has several limitations. All our participants spoke English, although many came from different cultures. Further research is necessary on whether non-English-speaking consumers have different views about chronic illness care. Our sample was skewed towards older people. The views of younger people with chronic illnesses also need to be investigated. Finally, two-thirds of our 12 groups (accounting for $77 \%$ of participants) were organised through consumer organisations. Such par- ticipants are more likely to be informed, articulate and actively involved in managing their condition. These factors reduce the generalisability of the results.

Some implications can be drawn from our findings. GPs might pay more attention to the time they spend with chronically ill patients, and to their interpersonal skills and understanding of patients' needs. They could endorse the contribution of patient self-management and consumer organisations and incorporate them into their care plans. GPs might also review their use of practice nurses and receptionists to maximise support for patients.

\section{ACKNOWLEDGEMENTS}

We thank the 76 focus group participants and $\mathrm{Mr}$ Frank Thaxton for an independent analysis of the transcripts. This study was funded by the Australian Department of Health and Ageing as part of a larger research study. The Department was not involved in the design or conduct of the study, and had no influence on the content of this article.

\section{COMPETING INTERESTS}

None identified.

\section{REFERENCES}

1 Mathers C, Vos T, Stevenson C. The burden of disease and injury in Australia. Canberra: Australian Institute of Health and Welfare; 1999. (Catalogue No. PHE 17.)

2 Carter S, Bonney M, Flack J, et al. National Divisions Diabetes Program Quality of Care and Health Outcomes: collated CARDIAB data. Sydney: Centre for General Practice Integration Studies, School of Public Health and Community Medicine, University of New South Wales; 2000.

3 Briganti EM, Shaw JS, Chadban SJ, et al. Untreated hypertension among Australian adults: the 1999-2000 Australian Diabetes, Obesity and Lifestyle Study (AusDiab). Med J Aust 2003; 179: 135-139.
4 Airey C, Erens B. National survey of NHS patients: general practice 1998. London: Department of Health; 2002.

5 Bower P, Roland M, Campbell J, Mead N. Setting standards based on patients' views on access and continuity: secondary analysis of data from the general practice assessment survey. BMJ 2003; 326: 258-262.

6 Grol R, Wensing M, Mainz J, et al. Patients in Europe evaluate general practice care: an international comparison. Br J Gen Pract 2000; 50: 882-887.

7 Consumers' Health Forum. Consumers' expectations of general practice in Australia. Canberra: Consumers' Health Forum, 1999.

8 Mott K. The consumer perspective. Med J Aust 2001; 175: 75-76.

9 Consumers' Health Forum. Integrating consumer views about quality in general practice. Canberra: AGPS, 1996.

10 Cheek J, Oster C. A qualitative investigation of the experiences, perceptions and understandings of people with a chronic condition. Adelaide: Centre for Research into Nursing and Health Care, University of Adelaide; 2002.

11 Oldroyd J, Proudfoot J, Infante F, et al. Providing healthcare for people with chronic illness: the views of Australian GPs. Med J Aust 2003; 179: 30-33.

12 Demirkol A, Richie J, Craig P. Providing healthcare for people with chronic illness: the views of Australian GPs [letter]. Med J Aust 2003; 179: 269.

13 NUD*IST NVivo [computer program]. Version 1.3.0. Melbourne: QSR International, 2000.

14 Martin C. Patient-practitioner relationship in chronic illness care. In: Walker C, Peterson CL, Millen N, Martin C, editors. Chronic illness: new perspectives and new directions. Melbourne: Tertiary Press, 2003.

15 Stewart M. Effective physician-patient communication and health outcomes: a review. CMAJ 1995; 152: 1423-1433.

16 Kamien M. The tasks of general practice - a new kind of GP? Aust Fam Physician 2002; 31: 1-3.

17 Tarrant C, Windridge K, Boulton M, et al. Qualitative study of the meaning of personal care in general practice. BMJ 2003; 326: 1310-1311.

(Received 16 Mar 2004, accepted 1 Jun 2004) $\square$ 